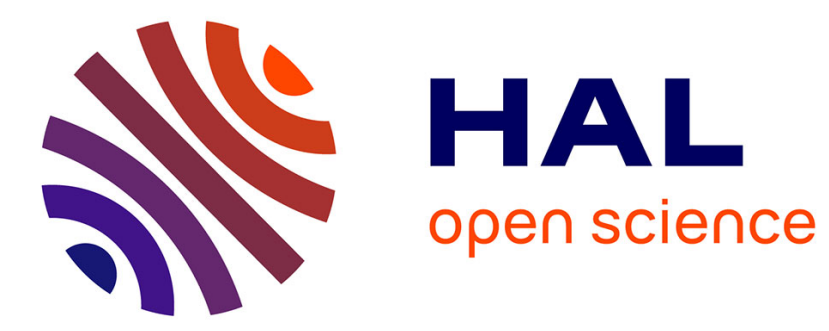

\title{
Variant of the truncated Perron formula and primes in polynomial sets
}

D. S Ramana, Olivier Ramaré

\section{To cite this version:}

D. S Ramana, Olivier Ramaré. Variant of the truncated Perron formula and primes in polynomial sets. International Journal of Number Theory, 2020, 16 (02), pp.309-323. 10.1142/S1793042120500165 . hal-02585974

\section{HAL Id: hal-02585974 https://hal.science/hal-02585974}

Submitted on 15 May 2020

HAL is a multi-disciplinary open access archive for the deposit and dissemination of scientific research documents, whether they are published or not. The documents may come from teaching and research institutions in France or abroad, or from public or private research centers.
L'archive ouverte pluridisciplinaire HAL, est destinée au dépôt et à la diffusion de documents scientifiques de niveau recherche, publiés ou non, émanant des établissements d'enseignement et de recherche français ou étrangers, des laboratoires publics ou privés. 
A Variant of the Truncated Perron's Formula and Primes in Polynomial Sets

\title{
VARIANT OF THE TRUNCATED PERRON FORMULA / PRIMES IN POLYNOMIAL SETS
}

\author{
D.S. RAMANA AND O. RAMARÉ
}

\begin{abstract}
We show under the Generalised Riemann Hypothesis that for every non-constant integer valued polynomial $f$, for every $\delta>0$, and almost every prime $q$ in $[Q, 2 Q]$, the number of primes from the interval $\left[x, x+x^{\frac{1}{2}+\delta}\right]$ that are values of $f$ modulo $q$ is the expected one, provided $Q$ is not more than $x^{\frac{2}{3}-\epsilon}$. We obtain this via a variant of the classical truncated Perron's formula for the partial sums of the coefficients of a Dirichlet series.
\end{abstract}

Perron's formula, GRH Primary 11N05; Secondary 11M06

\section{INTRODUCTION}

The classical truncated Perron's formula relates, for any $x \geq 1$, the partial sum $\sum_{1 \leq n \leq x} a_{n}$ of the coefficients of a Dirichlet series $F(s)=\sum_{n \geq 1} \frac{a_{n}}{n^{s}}$ with a finite abscissa of convergence $\sigma_{c}$ to the integral on the line segment $[\kappa-i T, \kappa+i T]$ of $\frac{F(s) x^{s}}{2 \pi i s}$, for any $T>0$ and $\kappa>\max \left(0, \sigma_{a}\right)$, where $\sigma_{a}$ is the abscissa of absolute convergence of $F(s)$. The difference between these two quantities is estimated by an error term that depends on a sum of the absolute values $\left|a_{n}\right|$ of the $a_{n}$. We present here a variant that has sums of the $a_{n}$ rather than $\left|a_{n}\right|$ and is valid for $\kappa>\max \left(0, \sigma_{c}\right)$. The basic version of this variant is stated in Theorem 2.1. This proposition results from a simple rewriting of the Fourier adjunction formula

$$
\int_{\mathbf{R}} f(u) \hat{\phi}(u) d u=\int_{\mathbf{R}} \hat{f}(u) \phi(u) d u
$$

valid for any $f, \phi$ in $\mathrm{L}^{1}(\mathbf{R})$, applied with $f(u)=e^{-\kappa u} \sum_{1 \leq n \leq x e^{u}} a_{n}$ and suitable $\phi$. Corollaries 2.2 puts Theorem 2.1 in applicable form. These are stated with the aid of notation introduced at the head of Section 2. At the end of this section we include a brief comparative description with other variants of the Perron formula in the literature such as those in G. Coppola \& S. Salerno [3], [2], J. Kaczorowski \& A. Perelli [6], J. Liu \& Y. Ye [7], and Wolke[8]. As an illustration of our version of the truncated Perron's formula, we shall obtain the following result in Section 3.

Theorem 1.1. Let $f$ be a non-constant integer valued polynomial. For any prime $q$, we set $V_{q}^{*}(f)$ to be the set of non zero values taken by $f$ modulo $q$. For any positive real numbers $Q \geq 2, \delta \leq 1 / 2$ and $\eta \leq 1 / 6$ and assuming the Generalised Riemann Hypothesis we have that for all but $o(Q / \log Q)$ primes $q$ in $[Q, 2 Q]$, the number of primes from $V_{q}^{*}(f)$ in $\left[x, x+x^{\frac{1}{2}+\delta}\right]$ is asymptotic to

$$
\frac{\left|V_{q}^{*}(f)\right|}{q-1} \frac{x^{\frac{1}{2}+\delta}}{\log x}
$$

Both authors has been partly supported by the Indo-French Centre for the Promotion of Advanced Research - CEFIPRA, project No 5401-1. The second author was supported by the EPSRC Grant EP/M50659X/1. 
provided that $x^{\frac{1}{6}+\delta-\eta} \geq Q$. Furthermore, for almost all prime moduli $q$ in $[Q, 2 Q]$, the sum $\sum_{p} \mu(p)$ where $p$ ranges the primes from $\left[x, x+x^{\frac{1}{2}+\delta}\right]$ that belong to $V_{q}^{*}(f)$ is $o\left(x^{\frac{1}{2}+\delta} / \log x\right)$ provided again that $x^{\frac{1}{6}+\delta-\eta} \geq Q$.

Note that it follows from a conjecture of Schur proved in [4] that the set $V_{q}(f)$ of values taken by $f$ modulo $q$ is infinitely often equal to the full $\mathbf{Z} / p \mathbf{Z}$ only for special polynomials. The authors do not know of any such result concerning $V_{q}^{*}(f)$.

In the final section of this note, Section 4, we consider the effect of "moving the line of integration $\sigma=\kappa "$ in the integrals on this line on the right hand sides of the formulae supplied by Corollary 2.2. In the classical case the kernel $\phi$ is identically equal to 1 on $[\kappa-i T, \kappa+i T]$. Our choices for $\phi$ are, however, sufficiently smooth, compactly supported, piecewise polynomial functions on $[\kappa-i T, \kappa+i T]$. These functions extend holomorphically in horizontal strips and, in general, these extensions are incompatible on adjacent strips. Nevertheless, Proposition 4.1 tells us that the smoothness of $\phi$ is enough to guarantee that the error due to this incompatibility on moving the line of integration is $O\left(x^{\kappa} / T^{2}\right)$ under reasonable assumptions on $F$.

Throughout this article we use $e(z)$ to denote $e^{2 \pi i z}$, for any complex number $z$. Further, all constants implied by the symbols $\ll$ and $\gg$ are absolute except when dependencies are indicated, either in words or by subscripts to these symbols. We will use the terms majorised and minorised to mean $\gg$ and $\ll$ respectively. The Fourier transform $\widehat{f}$ of an integrable function $f$ on $\mathbf{R}$ is defined by $\widehat{f}(u)=\int_{\mathbf{R}} f(t) e(-u t) d t$.

\section{The Variant}

Throughout this section, we let $F(s)=\sum_{n \geq 1} a_{n} / n^{s}$ be a Dirichlet series with a finite abscissa of convergence $\sigma_{c}$ and an abscissa of absolute convergence $\sigma_{a}$. Also, let $\sigma_{0}=\max \left(0, \sigma_{c}\right)$ and for any real $\sigma>\sigma_{0}$, let $B(\sigma)=\sup _{N \geq 1}\left|\sum_{1 \leq n \leq N} \frac{a_{n}}{n^{\sigma}}\right|$. Then on writing $a_{n}$ as $\frac{a_{n}}{n^{\sigma}} \cdot n^{\sigma}$ and using the Abel summation formula we obtain the classical bound of E. Cahen [1]:

$$
\left|\sum_{1 \leq n \leq x} a_{n}\right| \leq 2 B(\sigma) x^{\sigma}
$$

valid for all $x \geq 1$ and any $\sigma>\sigma_{0}$. The following theorem uses a test function $\phi$ and its Fourier transform $\hat{\phi}$ to express $\sum_{1 \leq n \leq x} a_{n}$ in terms of $F(s)$.

Theorem 2.1. Let $\phi$ a function in $L^{1}(\mathbf{R})$ with $\phi(0)=1$ and such that $\hat{\phi}$ is also in $L^{1}(\mathbf{R})$. Then for any $\kappa>\sigma_{0}$ and $x \geq 1$ we have

$$
\begin{aligned}
\sum_{1 \leq n \leq x} a_{n}=\frac{1}{2 \pi i} \int_{\kappa-i \infty}^{\kappa+i \infty} F(s) \phi & \left(\frac{s-\kappa}{2 \pi i}\right) \frac{x^{s}}{s} d s \\
+ & \int_{\mathbf{R}}\left(\sum_{1 \leq n \leq x} a_{n}-e^{-\kappa u} \sum_{1 \leq n \leq x e^{u}} a_{n}\right) \hat{\phi}(u) d u .
\end{aligned}
$$

Proof. For any $f$ in $\mathrm{L}^{1}(\mathbf{R})$ we have

$$
f(0)=\int_{\mathbf{R}} f(0) \hat{\phi}(u) d u=\int_{\mathbf{R}} \hat{f}(u) \phi(u) d u+\int_{\mathbf{R}}(f(0)-f(u)) \hat{\phi}(u) d u,
$$


on taking account of $\int_{\mathbf{R}} \hat{\phi}(u) d u=\phi(0)=1$ and (1), valid since $\phi$ and $\hat{\phi}$ are also in $\mathrm{L}^{1}(\mathbf{R})$. The relation (3) results on using (4) with

$$
f(u)=e^{-\kappa u} \sum_{1 \leq n \leq x e^{u}} a_{n}
$$

for the given $\kappa>\sigma_{0}$ and $x \geq 1$. Indeed, then $f(u)=0$ for $u<-\log x$. Further, we have from (2) with $\sigma$ in $\left(\sigma_{0}, \kappa\right)$ that $f$ is integrable in a neighbourhood of $+\infty$. Thus $f$ is in $\mathrm{L}^{1}(\mathbf{R})$. A comparision of the right hand side of (3) with the last term of (4) now shows that it only remains to verify that

$$
\hat{f}(u)=\frac{x^{s} F(s)}{s} \quad \text { where } s=\kappa+2 \pi i u,
$$

for all $u \in \mathbf{R}$, which is a well-known fact. For the sake of completeness, however, we provide a proof. For any integer $m \geq 1$, let $f_{m}(u)=e^{-\kappa u} \sum_{1 \leq n \leq m} a_{n} \chi_{n}(u)$, where $\chi_{n}(u)$ is 1 when $n \leq x e^{u}$ and is 0 otherwise. Then we certainly have $\lim _{m \rightarrow+\infty} f_{m}(u)=f(u)$ for all $u$ in $\mathbf{R}$. Also, (2) gives for any $\sigma$ in $\left(\sigma_{0}, \kappa\right)$ the bound

$$
\left|f_{m}(u)\right| \leq 2 B(\sigma) x^{\sigma} e^{(\sigma-\kappa) u}
$$

for all $m \geq 1$ and all $u$ in $\mathbf{R}$. This allows us to apply the dominated convergence theorem to justify the relation

$$
\begin{aligned}
\int_{\mathbf{R}} f(t) e^{-2 \pi i u t} d t & =\lim _{m \rightarrow+\infty} \int_{\mathbf{R}} f_{m}(t) e^{-2 \pi i u t} d t \\
& =\lim _{m \rightarrow+\infty} \sum_{1 \leq n \leq m} a_{n} \int_{\mathbf{R}} \chi_{n}(t) e^{-\kappa t} e^{-2 \pi i u t} d t
\end{aligned}
$$

for all $u$ in $\mathbf{R}$. Since $\kappa>0$ we have

$$
\int_{\mathbf{R}} \chi_{n}(t) e^{-\kappa t} e^{-2 \pi i u t} d t=\int_{\log \left(\frac{n}{x}\right)}^{\infty} e^{-(\kappa+2 \pi i u) t} d t=\frac{x^{s}}{s n^{s}}
$$

for all $n \geq 1$, where $s=\kappa+2 \pi i u$. Also, since $\kappa>\sigma_{c}$ we have $\lim _{m \rightarrow+\infty} \sum_{1 \leq n \leq m} \frac{a_{n}}{n^{s}}=$ $F(s)$. Consequently, (6) yields (5).

The following corollary puts the second term on the right hand side of (3) into a convenient form, with additional hypotheses on the test function $\phi$. These hypotheses are satisfied when $\phi$ is a sufficiently smooth positive compactly supported function with $\phi(0)=1$, as will be the case in our application.

Corollary 2.2. Let $\phi$ a function in $L^{1}(\mathbf{R})$ with $\phi(0)=1$ and such that

(i) $\hat{\phi}$ is in $L^{1}(\mathbf{R})$ and $\hat{\phi}(u)=\hat{\phi}(-u)$ for all $u$ in $\mathbf{R}$.

(ii) There is $m \geq 2$ such that $C_{k}(\phi)=\sup _{u \in \mathbf{R}}\left|u^{k} \hat{\phi}(u)\right|<+\infty$ for $0 \leq k \leq m+1$. 
We set $C(\phi)=\max _{0 \leq k \leq m+1} C_{k}(\phi)$. Then for any $\kappa>\sigma_{0}, x \geq 1$ and $T \geq 1$ we have

$$
\begin{aligned}
\sum_{1 \leq n \leq x} a_{n}= & \frac{1}{2 \pi i} \int_{\kappa-i \infty}^{\kappa+i \infty} F(s) \phi\left(\frac{s-\kappa}{2 \pi i T}\right) \frac{x^{s}}{s} d s \\
& +\int_{0}^{T}\left(\sum_{\left.x e^{-\frac{u}{T}<n \leq x e^{\frac{u}{T}}} a_{n} \operatorname{sgn}(x-n)\right) \hat{\phi}(u) d u}\right. \\
& +\mathcal{O}^{*}\left(\frac{4 C(\phi)(1+\kappa)^{2} e^{2 \kappa} B(\kappa) x^{\kappa}}{T}\right) .
\end{aligned}
$$

Proof. We first prove that

$$
\begin{aligned}
& \sum_{1 \leq n \leq x} a_{n}=\frac{1}{2 \pi i} \int_{\kappa-i \infty}^{\kappa+i \infty} F(s) \phi\left(\frac{s-\kappa}{2 \pi i T}\right) \frac{x^{s}}{s} d s \\
&+\int_{0}^{T}\left(\sum_{\left.x e^{-\frac{u}{T}<n \leq x e^{\frac{u}{T}}} a_{n} \operatorname{sgn}(x-n)\right)} \hat{\phi}(u) d u+\frac{\kappa}{T} \int_{0}^{T}\left(\sum_{x e^{-\frac{u}{T}<n \leq x e^{\frac{u}{T}}}} a_{n}\right) u \hat{\phi}(u) d u\right. \\
&+\mathcal{O}^{*}\left(\frac{4 C(\phi)\left(1+\kappa^{2}\right) e^{2 \kappa} B(\kappa) x^{\kappa} \log (e T)}{T^{2}}\right) .
\end{aligned}
$$

To do so, we use (4) with $f(u)=\phi(u / T)$ to obtain a similar version of (3). Plainly, the first terms on the right hand sides of (8) and (3) applied to $f$ are the same. If for the given $\kappa>\sigma_{0}$ and $x \geq 1$ we set $A(u)=\sum_{1 \leq n \leq x e^{u}} a_{n}$ for all $u \in \mathbf{R}$ then, since $\hat{f}$ is also even by $(i i)$, the second term on the right hand side of (3) applied to $f$ can be written

$$
\int_{0}^{+\infty}\left(2 A(0)-e^{-\kappa u} A(u)-e^{\kappa u} A(-u)\right) \hat{f}(u) d u .
$$

First we estimate the contribution to the integral (9) from the interval $[1,+\infty)$. From (2) with $\sigma=\kappa$ we see that $|A(0)|$ and $\left|e^{-\kappa u} A(u)\right|$ for $u \geq 1$ do not exceed $2 B(\kappa) x^{\kappa}$. Since $A(-u)=0$ when $u>\log x$, we similarly obtain $\left|e^{\kappa u} A(-u)\right| \leq$ $2 B(\kappa) x^{\kappa}$ for $u \geq 1$. Consequently, we have

$$
\begin{aligned}
& \left|\int_{1}^{+\infty}\left(2 A(0)-e^{-\kappa u} A(u)-e^{\kappa u} A(-u)\right) \hat{f}(u) d u\right| \\
& \quad \leq 8 B(\kappa) x^{\kappa} \int_{1}^{+\infty}|\hat{f}(u)| d u \leq 8 B(\kappa) x^{\kappa} C_{3}(f) \int_{1}^{+\infty} \frac{d u}{u^{3}} \leq 4 B(\kappa) x^{\kappa} C_{3}(f) .
\end{aligned}
$$

Let us now define $h(z)$ for any complex number $z$ by $e^{z}=1+z+h(z)$. Then the contribution to the integral (9) from the interval $(0,1)$ can be written as

$$
\begin{aligned}
& \int_{0}^{1}(2 A(0)-A(u)-A(-u)) \hat{f}(u) d u+\kappa \int_{0}^{1}(A(u)-A(-u)) u \hat{f}(u) d u \\
& -\int_{0}^{1}(h(-\kappa u) A(u)+h(\kappa u) A(-u)) \hat{f}(u) d u .
\end{aligned}
$$

We estimate the third integral in (11) by means of the bounds $|h(z)| \leq \frac{|z|^{2} e^{|z|}}{2}$ for all $z \in \mathbf{C}$ and $|A(u)| \leq 2 B(\kappa) x^{\kappa} e^{\kappa}$ for $|u| \leq 1$. The first of these bounds follows 
from the Taylor expansion of $e^{z}$ while the second follows from (2) with $\sigma=\kappa$. We obtain

$$
\begin{aligned}
\left|\int_{0}^{1}(h(-\kappa u) A(u)+h(\kappa u) A(-u)) \hat{f}(u) d u\right| & \\
& \leq 2 \kappa^{2} e^{2 \kappa} B(\kappa) x^{\kappa} \int_{0}^{1} u^{2}|\hat{f}(u)| d u .
\end{aligned}
$$

We have $\hat{f}(u)=T \hat{\phi}(u T)$ and therefore $C_{3}(f)=\frac{C_{3}(\phi)}{T}$ and $\int_{0}^{1} u^{2}|\hat{f}(u)| d u \leq \frac{C(\phi) \log (e T)}{T^{2}(11)}$. Also, on making the change of variable $u T \mapsto u$ in the first two integrals in (11) and recalling the definition of $A(u)$ we immediately see that these integrals are, respectively, the same as the second and third integrals on the right hand side of (8). Since $C_{3}(\phi) \leq C(\phi)$, the preceding remarks together with (10) and (12) gives (8).

Let us now simplify (8) further. Note that we have $|A(u)| \leq 2 B(\kappa) x^{\kappa} e^{\kappa}$ when $|u| \leq 1$ by (2). The triangle inequality gives

$$
\begin{aligned}
\frac{\kappa}{T}\left|\int_{0}^{T}\left(A\left(\frac{u}{T}\right)-A\left(-\frac{u}{T}\right)\right) u \hat{\phi}(u) d u\right| \leq \frac{4 \kappa B(\kappa) x^{\kappa} e^{\kappa}}{T} & \int_{0}^{T} u|\hat{\phi}(u)| d u \\
& \leq \frac{8 C(\phi) \kappa B(\kappa) x^{\kappa} e^{\kappa}}{T}
\end{aligned}
$$

since $\int_{0}^{T} u|\hat{\phi}(u)| d u \leq C_{0}(\phi)+C_{3}(\phi)$. By the definition of $A(u)$, the integrand in the first term of (13) is the same as that in the second integral on the right hand side of (8). Thus the corollary follows from the above estimate and (8), on noting that $\frac{\log (e T)}{T^{2}} \leq \frac{1}{T}$ when $T \geq 1$.

Remark 2.3. In basic applications it is useful to further simplify the second term on the right hand side of (7). Thus suppose that $\phi, T$ satisfy the conditions of the above corollary with $m=\ell+1, \ell \geq 1$ and let us for brevity set $E\left(\frac{u}{T}\right)=$ $\sum_{x e^{-\frac{u}{T}}<n \leq x e^{\frac{u}{T}}} a_{n} \operatorname{sgn}(x-n)$. Then on rewriting $E\left(\frac{u}{T}\right)$ as $2 A(0)-A\left(\frac{u}{T}\right)-A\left(-\frac{u}{T}\right)$ and using the Cahen bound (2) as above we get

$$
\int_{T^{1 / \ell}}^{T} E\left(\frac{u}{T}\right) \hat{\phi}(u) d u \ll_{\kappa, \phi} \frac{x^{\kappa}}{T}
$$

since $\int_{T^{1 / \ell}}^{T} u|\hat{\phi}(u)| d u \ll_{\phi} 1 / T$. Also, by the triangle inequality we have

$$
\int_{0}^{T^{1 / \ell}} E\left(\frac{u}{T}\right) d u \leq 2 C(\phi) \max _{0 \leq \xi \leq e T^{\frac{1}{\ell}-1}}\left(\left|\sum_{1<\frac{n}{x} \leq 1+\xi} a_{n}\right|+\left|\sum_{1 \leq \frac{x}{n}<1+\xi} a_{n}\right|\right),
$$

since $1+\xi=e^{\frac{u}{T}}$ implies $\xi \leq \frac{e u}{T}$ when $0 \leq u \leq T$, by the mean value theorem, and we have $\int_{0}^{T}|\hat{\phi}(u)| d u \leq C_{0}(\phi)+C_{2}(\phi)$. It follows from (14) and (15) that the sum of the second and third terms on the right hand side of (7) can be replaced with

$$
2 C(\phi) \max _{0 \leq \xi \leq e T^{\frac{1}{e}-1}}\left(\left|\sum_{1 \leq \frac{n}{x} \leq 1+\xi} a_{n}\right|+\left|\sum_{1 \leq \frac{x}{n} \leq 1+\xi} a_{n}\right|\right)+\mathcal{O}_{\kappa}\left(\frac{x^{\kappa}}{T}\right) .
$$

When used with a suitable $\phi$, for instance with $\phi=\mathfrak{p}_{3}(t ; 1)$ of $(41)$, Corollary 2.2 is of similar strength to Theorem 1 of Wolke [8]. The presence of the kernel $\phi$ dispenses with the delicate analysis required for the proof of Theorem 2 of [8]. Also, 
Corollary 2.2 merits comparison with Theorem 2.1 of Liu \& Ye [7]. In addition to the facts that (7) has sums of the $a_{n}$ rather than $\left|a_{n}\right|$ and is valid for $\kappa>\max \left(0, \sigma_{c}\right)$, we note that the error term in (7) has a $1 / T$ rather than essentially $1 / \sqrt{T}$ in Theorem 2.1 of [7].

It is perhaps pertinent here to remark that there is a small mistake in Theorem 1 of [8]: in inequality (2.5) therein, a factor $\left(\frac{x}{n}\right)^{\sigma}$ appears to be missing. This has the consequence that Theorem 2 of [8] is valid only for $T \geq \log x$, a restriction that is of no consequence for the applications. Theorem 1 of [6] must therefore also be read with the same restriction (A. Perelli agrees on this point) as it relies on [8].

One may hope to use the symmetry on account of the factor $\operatorname{sgn}(x-n)$ in the first error term of Proposition 2.2. This is undoubtedly very difficult in general, but see Coppola and Salerno [3] and [2] for a treatment. Theorem 1 in Kaczorowski \& Perelli [6] also gives a formula with a similar symmetry.

Thanks.

\section{Proof of the Theorem}

We start with a non-constant integer valued polynomial $f$. For any prime $q$, we define $V_{q}^{*}(f)$ to be the set of non zero values taken by $f$ modulo $q$.

If for any integer $n$ we write $\tilde{n}$ to denote the image of $n$ modulo $q$ and write $1_{V_{q}^{*}(f)}$ for the characteristic function of $V_{q}^{*}(f)$, then we have that

$$
1_{V_{q}^{*}(f)}(\tilde{n})=\sum_{\chi \bmod q} c_{q}(\chi) \chi(n)
$$

for all integers $n$, where the sum runs over all Dirichlet characters $\chi$ modulo $q$ with $c_{q}(\chi)$ defined to be $\frac{1}{\phi(q)} \sum_{a \in V_{q}^{*}(f)} \overline{\chi(a)}$. To quantify our estimates, we use

$$
W_{1}=\sum_{\substack{Q \leq q \leq 2 Q, q \text { prime }}} \sum_{\substack{\bmod q, \chi \neq \chi_{0}}}\left|c_{q}(\chi)\right|, \quad W_{2}=\sum_{\substack{Q \leq q \leq 2 Q, q \text { prime }}} \sum_{\substack{\bmod q, \chi \neq \chi_{0}}}\left|c_{q}(\chi)\right|^{2} .
$$

By an application of the Cauchy-Schwarz inequality followed by the Parseval relation for the group $(\mathbf{Z} / q \mathbf{Z})^{*}$ we get

$$
\sum_{\chi \bmod q}\left|c_{q}(\chi)\right| \leq \phi(q)^{\frac{1}{2}}\left(\sum_{\chi \bmod q}|c(\chi)|^{2}\right)^{\frac{1}{2}}=\left|V_{q}^{*}(f)\right|^{\frac{1}{2}}
$$

while $\sum_{\substack{\chi \neq \chi_{0} \\ \bmod q}}\left|c_{q}(\chi)\right|^{2} \leq\left|V_{q}^{*}(f)\right| / \phi(q)$ by the Parseval relation. Since the polynomial $f$ is integer-valued, it is an integral linear combination of binomial polynomials, from which we deduce that there exists an integer $b$ such that $c f$ is polynomial in $\mathbf{Z}[X]$. A further consequence is that the equation $f(x)=m$ modulo the prime $q$ has, except for finitely many $q$ 's, at most the degree of $f$ solutions; indeed, we only have to avoid those $q$ 's for which $c f$ modulo $q$ becomes a constant polynomial. We conclude from these remarks that, except for finitely many primes, we have $1 / \operatorname{deg}(f) \leq\left|V_{q}^{*}(f)\right| / \phi(q) \leq 1$. We will however refrain to use these bounds until (31) and only use $W_{1}$ and $W_{2}$. 
Throughout the remainder of this section $\mathbf{b}=\left\{b_{n}\right\}_{n \geq 1}$ will denote one of the sequences $\{\Lambda(n)\}_{n \geq 1}$ and $\{\mu(n)\}_{n \geq 1}$. Then for any real number $w \geq 1$ we have

$$
\sum_{\substack{Q \leq q \leq 2 Q, q \text { prime }}} \sum_{\substack{1 \leq n \leq w, n \in V_{q}^{*}(f) \bmod q .}} b_{n}=\sum_{\substack{Q \leq q \leq 2 Q, q \text { prime }}} \sum_{\chi \bmod q} c_{q}(\chi) \sum_{1 \leq n \leq w} \chi(n) b_{n} .
$$

For a given real $x \geq 1$, let us set $y=x^{\theta}$ with $0<\theta<1$. Then on subtracting the contribution from the principal character $\chi_{0}$ modulo $q$ to the right hand side of (20) from both sides of this relation and using the resulting relation for $w=x$, $w=x+y$ together with triangle inequality we get

$$
\begin{aligned}
\sum_{\substack{Q \leq q \leq 2 Q, q \text { prime }}}\left|\sum_{\substack{x<n \leq x+y, n \in V_{q}^{*}(f) \bmod q .}} b_{n}-\frac{\left|V_{q}^{*}(f)\right|}{\phi(q)} \sum_{\substack{x<n \leq x+y,(n, q)=1 .}} b_{n}\right| \\
\leq \sum_{\substack{Q \leq q \leq 2 Q, q \text { prime }}}\left|\sum_{\substack{\chi \bmod q, \chi \neq \chi_{0} .}} c_{q}(\chi) \sum_{x<n \leq x+y} \chi(n) b_{n}\right|
\end{aligned}
$$

We shall presently bound the sum

$$
\Sigma=\Sigma(\mathbf{b}, x, y, Q)=\sum_{\substack{Q \leq q \leq 2 Q, q \text { prime }}}\left|\sum_{\substack{\chi \bmod q, \chi \neq \chi_{0} .}} c_{q}(\chi) \sum_{x<n \leq x+y} b_{n} \chi(n)\right| .
$$

by means of Corollary 2.2. To this end, we set $F(s, \chi)=\sum_{n \geq 1} b_{n} \chi(n) / n^{s}$, which converges in $\sigma>\frac{1}{2}$ for each $\chi \neq \chi_{0}$ under the GRH for our sequences $\mathbf{b}$. We then fix a $\epsilon>0$ and set $\kappa=\frac{1}{2}+\epsilon$. Also, we let $\varphi$ be a positive continuous function supported in $[-1,1]$ and satisfying the conditions on $\phi$ of Corollary 2.2 with $m=2$. For example we may take $\phi=\mathfrak{p}_{3}(t ; 1)$ of $(41)$. On applying this corollary we now get

$$
\begin{aligned}
\sum_{1 \leq n \leq w} b_{n} \chi(n) & =\frac{1}{2 \pi i} \int_{\kappa-i \infty}^{\kappa+i \infty} F(s, \chi) \varphi\left(\frac{s-\kappa}{2 \pi i T}\right) \frac{w^{s}}{s} d s \\
& +\int_{0}^{T}\left(\sum_{w e^{-\frac{u}{T}<n \leq w e^{\frac{u}{T}}}} b_{n} \chi(n) \operatorname{sgn}(w-n)\right) \hat{\varphi}(u) d u \\
& +O\left(\frac{B(\kappa, \chi) w^{\kappa} \log (e T)}{T^{2}}\right),
\end{aligned}
$$

for all real numbers $w \geq 1, T \geq 1$ and $\chi \neq \chi_{0}$. Here we have $B(\kappa, \chi)=$ $\sup _{N \geq 1}\left|\sum_{1 \leq n \leq N} \frac{b_{n} \chi(n)}{n^{\kappa}}\right| \ll_{\epsilon} q^{\epsilon}$ under the GRH for sequences $\mathbf{b}$ given above, as can be seen by integrating by parts using Theorem 15.5 of [5]. We now note the following lemma, which allows us to take advantage of the cancellation in the sums on the right hand side of the above relation. 
Lemma 3.1. Let $w \geq T \geq 1$ be two real numbers. Then with $\left\{b_{n}\right\}_{n \geq 1}$ as above, we have

$$
\begin{array}{r}
\int_{0}^{T} \sum_{\substack{Q \leq q \leq 2 Q, q \text { prime }}}\left|\sum_{\substack{\chi \bmod q, \chi \neq \chi_{0} .}} c_{q}(\chi) \sum_{e^{-\frac{u}{T}}<n \leq w e^{\frac{u}{T}}} b_{n} \operatorname{sgn}(w-n) \chi(n) \hat{\varphi}(u)\right| d u \\
\ll \max _{1 \leq n \leq e w}\left|b_{n}\right|\left(\frac{w \log (e T)}{T}+\frac{\sqrt{w}}{\sqrt{T}} Q\right) \sqrt{W_{2}} .
\end{array}
$$

where the implied constant depends on $\varphi$ alone.

Proof. We set $u_{n}=b_{n} \operatorname{sgn}(w-n)$. By means of the triangle inequality and the Cauchy-Schwarz inequality we have

$$
\begin{aligned}
& \left(\int_{0}^{T} \sum_{\substack{Q \leq q \leq 2 Q \\
q \text { prime }}}\left|\sum_{\substack{\chi \bmod q, \chi \neq \chi_{0} .}} c_{q}(\chi) \sum_{w e^{-\frac{u}{T}<n \leq w e^{\frac{u}{T}}}} u_{n} \chi(n) \hat{\varphi}(u)\right| d u\right)^{2} \\
& \leq\left(\sum_{\substack{Q \leq q \leq 2 Q, q \text { prime }}} \sum_{\substack{\bmod q, \chi \neq \chi_{0} .}}\left|c_{q}(\chi)\right|^{2} \int_{0}^{T}|\hat{\varphi}(u)| d u\right) \times \\
& \left(\left.\int_{0}^{T} \sum_{\substack{Q \leq q \leq 2 Q, q \operatorname{prime}}} \sum_{\substack{\bmod q, \chi \neq \chi_{0} .}} \sum_{w e^{-\frac{u}{T}<n \leq w e^{\frac{u}{T}}}} u_{n} \chi(n)\right|^{2}|\hat{\varphi}(u)| d u\right) .
\end{aligned}
$$

Since $\hat{\varphi}$ is integrable on $\mathbf{R}$, the first of the two bracketed expressions on the right hand side of the above relation does not exceed $\|\hat{\varphi}\|_{1} W_{1}$. We estimate the second expression using the variant of the large sieve inequality for characters that can be found for instance in [5, Theorem 7.13]. Indeed, when $w \geq 1$ and $u>0$, the number of integers in $\left(w e^{-\frac{u}{T}}, w e^{\frac{u}{T}}\right]$ is at most $\frac{2 w u e^{\frac{u}{T}}}{T}+1$. Then it follows from this inequality that

$$
\begin{aligned}
& \sum_{\substack{Q \leq q \leq 2 Q, \chi \\
q \text { prime }}} \sum_{\substack{\text { mod } \\
\chi \neq \chi_{0}}}\left|\sum_{w e^{-\frac{u}{T}<n \leq w e^{\frac{u}{T}}}} u_{n} \chi(n)\right|^{2} \\
& \quad \leq \max _{w e^{-\frac{u}{T}<n \leq w e^{\frac{u}{T}}}}\left|u_{n}\right|^{2}\left(\frac{2 w u e^{\frac{u}{T}}}{T}+1\right)\left(\frac{2 w u e^{\frac{u}{T}}}{T}+4 Q^{2}\right) \\
& \ll \max _{w e^{-\frac{u}{T}}<n \leq w e^{\frac{u}{T}}}\left|u_{n}\right|^{2}\left(\frac{w^{2} u^{2}}{T^{2}}+\frac{w u}{T} Q^{2}+Q^{2}\right) .
\end{aligned}
$$

On noting that $\int_{0}^{T} u^{2}|\hat{\varphi}(u)| d u \leq C(\phi) \log (e T), \int_{0}^{T} u|\hat{\varphi}(u)| d u \leq 2 C(\phi)$ and $\int_{0}^{T}|\hat{\varphi}(u)| d u \leq 2 C(\phi)$ we conclude that the second expression in the brackets on the right hand side of (25) is majorised by

$$
\begin{aligned}
\max _{1 \leq n \leq e x}\left|u_{n}\right|^{2} \int_{0}^{T}\left(\frac{w^{2} u^{2}}{T^{2}}+\frac{w u}{T} Q^{2}\right. & \left.+Q^{2}\right)|\hat{\varphi}(u)| d u \\
& \ll \max _{1 \leq n \leq e w}\left|u_{n}\right|^{2}\left(\frac{w^{2} \log (e T)}{T^{2}}+\frac{w}{T} Q^{2}+Q^{2}\right) .
\end{aligned}
$$

Since $T \leq w$, the lemma now follows on substituting the preceding bounds into (25) and passing to square roots. 
We sum the absolute values of both sides of (23) over the characters $\chi \neq \chi_{0}$ and the primes $q$ in $[Q, 2 Q]$. We then estimate the second and third terms on the right hand side of the resulting relation using Lemma 3.1. On using (19) to bound the error term of this relation we conclude that

$$
\begin{aligned}
\sum_{\substack{Q \leq q \leq 2 Q \\
q \text { prime }}} \sum_{\substack{\chi \bmod q, 1 \leq n \leq w \\
\chi \neq \chi_{0} .}} \sum_{n} \chi(n) & =\frac{1}{2 \pi i} \int_{\kappa-i \infty}^{\kappa+i \infty} \sum_{\substack{Q \leq q \leq 2 Q, \chi \\
q \text { prime }}} \sum_{\substack{\chi \neq \chi_{0} . \\
\text { mod } q}} F(s, \chi) \varphi\left(\frac{s-\kappa}{2 \pi i T}\right) \frac{w^{s}}{s} d s \\
& +O\left(\max _{1 \leq n \leq e w}\left|b_{n}\right|\left(\frac{w \log (e T)}{T}+\frac{\sqrt{w}}{\sqrt{T}} Q\right) \sqrt{W_{2}}\right) \\
& +O_{\epsilon}\left(\frac{Q^{\epsilon} \log (e T) w^{\frac{1}{2}+\epsilon} W_{1}}{T^{2}}\right)
\end{aligned}
$$

for all real $w \geq 1$. We apply this with $w=x$ and $w=x+y$, subtract and recall the definition of $\Sigma$ to obtain by means of the triangle inequality that

$$
\begin{aligned}
\Sigma & \leq \frac{1}{2 \pi} \int_{\kappa-i \infty}^{\kappa+i \infty} \sum_{\substack{Q \leq q \leq 2 Q, q \text { prime }}} \sum_{\substack{\chi \neq \chi_{0} . \\
\chi \neq 0}}\left|c_{q}(\chi)\right||F(s, \chi)|\left|\varphi\left(\frac{s-\kappa}{2 \pi i T}\right)\right|\left|\frac{(x+y)^{s}-x^{s}}{s}\right| d t \\
& +O\left(\max _{1 \leq n \leq 2 e x}\left|b_{n}\right|\left(\frac{x \log (e T)}{T}+\frac{\sqrt{x}}{\sqrt{T}} Q\right) \sqrt{W_{2}}\right)+O_{\epsilon}\left(\frac{W_{1} x^{\frac{1}{2}+\epsilon} \log (e T)}{T^{2}}\right) .
\end{aligned}
$$

On the GRH we have the classical Lindelöf bound $|F(s, \chi)| \ll_{\epsilon}(q+q|t|)^{\epsilon}$, by [5], Theorem 5.17 and Corollary 5.19. Also, for $s=\kappa+i t$ we have $\left|\frac{(x+y)^{s}-x^{s}}{s}\right| \leq$ $\min \left(\frac{3 x^{\kappa}}{|s|}, x^{\kappa-1} y\right)$ by a trivial estimate and the mean value theorem. Further, $\varphi\left(\frac{s-\kappa}{2 \pi i T}\right)=$ 0 when $|t| \geq T$. On combining these remarks with (19) and assuming that $T \leq x$, we see that

$$
\begin{aligned}
& \int_{\kappa-i \infty}^{\kappa+i \infty} \sum_{\substack{Q \leq q \leq 2 Q, q \text { prime }}} \sum_{\substack{\bmod q, \chi \neq \chi_{0}}}\left|c_{q}(\chi)\right||F(s, \chi)| \varphi\left(\frac{s-\kappa}{2 \pi i T}\right)|| \frac{(x+y)^{s}-x^{s}}{s} \mid d t \\
& \ll_{\epsilon} W_{1}(x Q)^{\epsilon} \int_{-T}^{T} \min \left(\frac{3 x^{\kappa}}{|\kappa+i t|}, x^{\kappa-1} y\right) d t \ll_{\epsilon} \frac{y W_{1}}{x^{\frac{1}{2}}} \min \left(T, \frac{x}{y}\right)(x Q)^{\epsilon}
\end{aligned}
$$

Using this in $(24)$ and noting that $\log (e T) \ll_{\epsilon} x^{\epsilon}$ we finally obtain

$$
\Sigma \ll_{\epsilon}(x Q)^{\epsilon}\left(\frac{y W_{1}}{x^{\frac{1}{2}}} \min \left(T, \frac{x}{y}\right)+\frac{x \sqrt{W_{2}}}{T}+\frac{Q \sqrt{x W_{2}}}{T^{1 / 2}}\right),
$$

since for our choices of the sequence $\mathbf{b}$ we certainly have $\max _{1 \leq n \leq 2 e x}\left|b_{n}\right| \ll_{\epsilon} x^{\epsilon}$. If we select $T$ larger than $x / y$, then the first term of our majorant is $O\left((x Q)^{\epsilon} W_{1} \sqrt{x}\right)$, which is what using the GRH of each sum modulo $q$ gives. Since we want to improve on this bound, we seek to choose $T$ smaller. We set $T=x^{3 / 4} W_{2}^{1 / 4} / \sqrt{y W_{1}}$ which we assume to be within $[1, x]$. Then on combining (30) with (21) and (22) we get 
the bound

$(31)$

$\sum_{\substack{Q \leq q \leq 2 Q, q \text { prime }}}\left|\sum_{\substack{x<n \leq x+y, n \in V_{q}^{*}(f)}} b_{n}-\frac{\left|V_{q}^{*}(f)\right|}{\phi(q)} \sum_{\substack{x<n \leq x+y,(n, q)=1 .}} b_{n}\right| \ll_{\epsilon} y Q(Q x)^{\epsilon} x^{1 / 4}\left(\frac{W_{2}^{1 / 4} W_{1}^{1 / 2}}{y^{1 / 2} Q}+\frac{W_{2}^{3 / 8} W_{1}^{1 / 4}}{y^{3 / 4}}\right)$.

We now employ the bounds $W_{1} \ll Q^{3 / 2}$ and $W_{2} \ll Q$ to infer that

$$
\begin{gathered}
\sum_{\substack{Q \leq q \leq 2 Q, q \text { prime }}}\left|\sum_{\substack{x<n \leq x+y, n \in V_{q}^{*}(f)}} b_{n}-\frac{\left|V_{q}^{*}(f)\right|}{\phi(q)} \sum_{\substack{x<n \leq x+y,(n, q)=1 .}} b_{n}\right| \\
\ll_{\epsilon} y Q(Q x)^{\epsilon} x^{1 / 4}\left(\frac{1}{y^{1 / 2}}+\frac{Q^{3 / 4}}{y^{3 / 4}}\right) .
\end{gathered}
$$

The assumed condition $T \geq 2$ is granted by the condition $Q y \leq c x^{3 / 2}$ for a large enough constant $c$.

3.1. The case of the primes. We now take $b_{n}=\Lambda(n)$ in (32) and verify the first conclusion of Theorem 1.1. In effect, since $Q \leq x$, in this case (32) can be rewritten as

$$
\frac{\log Q}{Q} \sum_{\substack{Q \leq q \leq 2 Q, q \text { prime }}}\left|\sum_{\substack{x<n \leq x+y, n \in V_{q}^{*}(f)}} \Lambda(n)-\frac{\left|V_{q}^{*}(f)\right|}{\phi(q)} \sum_{\substack{x<n \leq x+y,(n, q)=1 .}} \Lambda(n)\right| \ll_{\epsilon} y x^{\epsilon}\left(\frac{x^{1 / 4}}{y^{1 / 2}}+\frac{Q^{3 / 4} x^{1 / 4}}{y^{3 / 4}}\right) .
$$

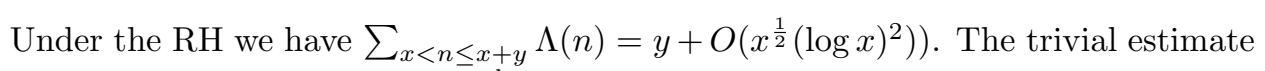
for the contribution from $n=p^{k}$, with $p$ prime and $k \geq 2$, to the sums inside the absolute value on the left hand side is $O\left(x^{\frac{1}{2}}(\log x)^{2}\right)$. Since $Q \leq x$, the condition $(n, q)=1$ on the left hand side can be dropped when $n$ is a prime. These remarks yield

$$
\frac{\log Q}{Q} \sum_{\substack{Q \leq q \leq 2 Q, q \text { prime. }}}\left|\sum_{\substack{x<p \leq x+y, p \in V_{q}^{*}(f) \\ p \text { prime. }}} \log p-\frac{\left|V_{q}^{*}(f)\right| y}{\phi(q)}\right| \ll_{\epsilon} y(Q x)^{\epsilon}\left(\frac{x^{1 / 4}}{y^{1 / 2}}+\frac{Q^{3 / 4} x^{1 / 4}}{y^{3 / 4}}\right)+x^{\frac{1}{2}}(\log x)^{2} .
$$

With $y=x^{\frac{1}{2}+\delta}$ we have $x^{\frac{1}{2}}(\log x)^{2} \ll y x^{\epsilon} x^{-\frac{\delta}{2}}$. Since also $Q \leq x^{\theta}$ with $\theta=\delta+\frac{1}{6}-\eta$, we get

$$
\frac{\log Q}{Q} \sum_{\substack{Q \leq q \leq 2 Q, q \text { prime. }}}\left|\sum_{\substack{x<p \leq x+y, p \in V_{q}^{*}(f) \\ p \text { prime. }}} \log p-\frac{\left|V_{q}^{*}(f)\right| y}{\phi(q)}\right| \ll_{\epsilon} y x^{\epsilon}\left(x^{-\delta / 2}+x^{-3 \eta / 4}\right) .
$$

As noted before, for all but finitely many prime numbers $q$ we have $\left|V_{q}^{*}(f)\right| \geq$ $(p-1) / \operatorname{deg} f$ and $\phi(q)=q-1$. Thus if $\mathcal{S}$ is the set of primes $q$ in $[Q, 2 Q]$ such that

$$
\left|\sum_{\substack{x-y \leq p \leq x, p \in V_{q}^{*}(f)}} \log p-\frac{\left|V_{q}^{*}(f)\right| y}{q-1}\right| \geq \frac{\left|V_{q}^{*}(f)\right| y}{q-1} x^{-\xi}, \quad \xi=\min (\delta / 2,3 \eta / 4)
$$


then it follows from (33) with $\epsilon=\xi / 2$ that

$$
\frac{|\mathcal{S}|}{Q} \ll x^{-\xi / 2} \ll Q^{-\xi / 2},
$$

which yields the desired conclusion of the theorem after removing the weights $\log p$ in the usual fashion.

3.2. The case of the Möbius function. Here we set $b_{n}=\mu(n)$ in (32) and carry out the details just as in the preceding case, taking note of the simplification afforded by the fact that in this case there is no main term and no prime powers in the support of the function $\mu$.

\section{Moving the Line of Integration}

Our first purpose here is to record the proposition below that describes the effect of "moving the line of integration" in the integrals over the line $\sigma=\kappa$ on the right hand sides of (8) and (7) when $\phi$ is a given continuous positive compactly supported piecewise polynomial function.

It will be convenient here to use both $s=\sigma+i t$ and $z=u+i v$ to denote complex numbers. Also, we shall suppose that the support of $\phi$ is in $[-U, U]$ for some $U>0$. Further, let $-U=u_{1}<u_{2}<\ldots<u_{m}=U$ be such that the restriction of $\phi$ to the real interval $\left[u_{j}, u_{j+1}\right)$ agrees with that of a polynomial $\widetilde{\phi}_{j}$ defined on $\mathbf{C}$, for $1 \leq j \leq m-1$. We will assume that $u_{j} \neq 0$ and let $\eta \leq\left|u_{j}\right|$ for all $j$. Let $V>0$ be a positive real number and let $M$ satisfy $\left|\widetilde{\phi}_{j}^{\prime}(z)\right| \leq M$ for all $z=u+i v$ in the rectangle $-U \leq u \leq U$ and $0 \leq v \leq V$ and $1 \leq j \leq m-1$. Finally, we define $\widetilde{\phi}(z)$ for $z=u+i v$ with $u \in[-U, U)$ by $\widetilde{\phi}(z)=\widetilde{\phi}_{j}(z)$ where $j$ is the unique index such that $u \in\left[u_{j}, u_{j+1}\right)$.

Proposition 4.1. With notation as above, let $\kappa^{\prime}, \kappa$ be such that $0<\kappa-\kappa^{\prime} \leq 2 \pi V$. Also, let $F$ be a meromorphic function on a neighbourhood of the closed rectangle with vertices $\kappa^{\prime} \pm 2 \pi i U T$ and $\kappa \pm 2 \pi i U T$, for some $T \geq 1$. Suppose further that if $\mathcal{A}$ is the set of poles of $s \mapsto F(s) / s$ in this neighbourhood then $\operatorname{Re}(a) \neq \kappa, \kappa^{\prime}$ and $\operatorname{Im}(a) \neq 2 \pi u_{j}$ for all $a$ in $\mathcal{A}$ and $1 \leq j \leq m$. Then we have that

$$
\begin{aligned}
\frac{1}{2 \pi i} \int_{\kappa-i \infty}^{\kappa+i \infty} F(s) \phi & \left.\frac{s-\kappa}{2 \pi i T}\right) \frac{x^{s}}{s} d s=\frac{1}{2 \pi i} \int_{\kappa^{\prime}-i \infty}^{\kappa^{\prime}+i \infty} F(s) \widetilde{\phi}\left(\frac{s-\kappa}{2 \pi i T}\right) \frac{x^{s}}{s} d s \\
& +\sum_{a \in \mathcal{A}} \operatorname{Res}\left(\frac{\widetilde{\phi}(s) F(s) x^{s}}{s}\right)_{s=a} \\
& +\mathcal{O}^{*}\left(\frac{M\left(\kappa-\kappa^{\prime}\right) x^{\kappa}}{4 \pi^{3} \eta T^{2}} \sum_{1 \leq j \leq m} \int_{\kappa}^{\kappa^{\prime}}\left|F\left(\sigma+2 \pi i u_{j} T\right)\right| d \sigma\right) .
\end{aligned}
$$

Proof. For $1 \leq j \leq m-1$, the function $G$ with $G(s)=\frac{F(s) \widetilde{\phi}_{j}\left(\frac{s-\kappa}{2 \pi i T}\right) x^{s}}{s}$ is meromorphic in a neighbourhood of the closed rectangle $\mathcal{R}_{j}$ with vertices $\kappa^{\prime}+2 \pi i u_{j+1} T$, $\kappa^{\prime}+2 \pi i u_{j} T$ and $\kappa+2 \pi i u_{j} T, \kappa+2 \pi i u_{j+1} T$, with no poles on the boundary of this rectangle. On applying the residue theorem to $G$ on each $\mathcal{R}_{j}$ oriented anticlockwise for $1 \leq j \leq m-1$ and adding the resulting relations, we see that (36) follows if we show that the sum of the integrals of $G$ along the oriented horizontal sides of the 
$\mathcal{R}_{j}$ is majorised by the error term in (36). This reduces to verifying for $1 \leq j \leq m$ the inequality

$$
\left|\frac{1}{2 \pi i} \int_{\kappa^{\prime}+2 \pi i u_{j} T}^{\kappa^{\prime}+2 \pi i u_{j} T} F(s) x^{s} P_{j}\left(\frac{s-\kappa}{2 \pi i T}\right) \frac{d s}{s}\right| \leq \frac{M\left(\kappa-\kappa^{\prime}\right) x^{\kappa}}{4 \pi^{2} \eta T^{2}} \int_{\kappa}^{\kappa^{\prime}}\left|F\left(\sigma+2 \pi i u_{j} T\right)\right| d \sigma,
$$

where $P_{1}(s)=\widetilde{\phi}_{1}(s), P_{m}(s)=\widetilde{\phi}_{m-1}(s)$ and $P_{j}(s)=\widetilde{\phi}_{j}(s)-\widetilde{\phi}_{j-1}(s)$ for $2 \leq j \leq$ $m-1$. For $1 \leq j \leq m$ and $\sigma \in\left[\kappa^{\prime}, \kappa\right]$, let us set $a_{j}(\sigma)=P_{j}\left(\frac{\sigma+2 \pi i u_{j} T-\kappa}{2 \pi i T}\right)$. Then since $\phi$ is continuous and supported in $[-U, U]$, we have $a_{j}(\kappa)=P_{j}\left(u_{j}\right)=0$ for each $j$. Thus the mean value theorem applied to $\sigma \mapsto a_{j}(\sigma)$, which is a continuously differentiable function, gives

$$
\left|P_{j}\left(\frac{\sigma+2 \pi i u_{j} T-\kappa}{2 \pi i T}\right)\right|=\left|a_{j}(\sigma)-a_{j}(\kappa)\right| \leq \frac{2 M\left(\kappa-\kappa^{\prime}\right)}{2 \pi T},
$$

for all $\sigma \in\left[\kappa^{\prime}, \kappa\right]$ and $1 \leq j \leq m$. Here we have used $0 \leq \frac{\kappa-\kappa^{\prime}}{2 \pi T} \leq V$, since $T \geq 1$. Also, for $\sigma \in\left[\kappa^{\prime}, \kappa\right]$ we have $\left|x^{\sigma+2 \pi i u_{j} T}\right| \leq x^{\kappa}$ and $\left|\sigma+2 \pi i u_{j} T\right| \geq 2 \pi \eta T$ for $1 \leq j \leq m$. These bounds together with an application of the triangle inequality to the left hand side of (37) verify this inequality.

We now describe a convenient family test functions that may be used for $\phi$ in our formulae. Let us we set $\delta>0$ and $m \geq 1$, an integer. Also, we will write $1_{[a, b]}$ for the characteristic function of the interval $[a, b]$ and $1_{[a, b]}^{(* m)}$ for the $m$-th convolution of $1_{[a, b]}$ with iteslf. Then we define even function $\mathfrak{p}_{m}(t ; \delta)$ by

$$
\mathfrak{p}_{m}(t ; \delta)=\int_{-\infty}^{\infty} 1_{[-1,1]}\left(\frac{u}{1+\frac{1}{2} \delta}\right) 1_{[-1,1]}^{(* m)}\left(\frac{t-u}{\frac{1}{2} \delta / m}\right) \frac{m d u}{2^{m} \frac{1}{2} \delta} .
$$

It is easily seen that $\mathfrak{p}_{m}(t ; \delta)$ is piecewise polynomial of class $C^{m}$ and that its support lies in $[-(1+\delta), 1+\delta]$. Moreover, we have $\mathfrak{p}_{m}(t ; \delta)=1$ when $|t| \leq 1$ and $0 \leq \mathfrak{p}_{m}(t ; \delta) \leq 1$ for all real $t$. Finally, it immediately follows from basic properties of the Fourier transform that

$$
\hat{\mathfrak{p}}_{m}(u ; \delta)=\frac{\sin (\pi(2+\delta) u)}{\pi u}\left(\frac{m \sin (\pi \delta u / m)}{\pi \delta u}\right)^{m}
$$

and that $\hat{\mathfrak{p}}_{m}(-u ; \delta)=\hat{\mathfrak{p}}_{m}(u ; \delta)$. We end this note by explicitly describing $\mathfrak{p}_{3}(t ; 1)$ :

$$
\mathfrak{p}_{3}(t ; 1)= \begin{cases}0 & \text { when }|t| \geq 3 / 2, \\ 3(3-2 t)^{3} / 16 & \text { when } 7 / 6 \leq|t| \leq 3 / 2 \\ \left(36 t^{3}-108 t^{2}+99 t-25\right) / 4 & \text { when } 5 / 6 \leq|t| \leq 7 / 6 \\ \left(-9 t^{3}+27 t^{2}-27 t+25\right) / 16 & \text { when } 1 / 2 \leq|t| \leq 5 / 6 \\ 1 & \text { when }|t| \leq 1 / 2\end{cases}
$$

Acknowledgement : The authors are grateful to Hervé Queffelec for the many interesting discussions on Fourier and Mellin transforms and Perron's formula. Thanks are also due to Eero Saskman for sharing his ideas and to the referee for his/her vigilance while reading of the manuscript. This work was put in final form with support from the CEFIPRA project 5401-1. The first author gratefully acknowledges the facilities provided to him by the Université Aix-Marseille during his visit under the aegis of the said project. 


\section{REFERENCES}

[1] E. Cahen. Sur la fonction $\zeta(s)$ de Riemann et sur des fonctions analogues. 1894. http://www. numdam.org/item?id=ASENS_1894_3_11__75_0.

[2] G. Coppola and S. Salerno. On the symmetry of arithmetical functions in almost all short intervals. C. R. Math. Acad. Sci. Soc. R. Can., 26(4):118-125, 2004.

[3] G. Coppola and S. Salerno. On the symmetry of the divisor function in almost all short intervals. Acta Arith., 113(2), 2004.

[4] Michael Fried. On a conjecture of Schur. Michigan Math. J., 17:41-55, 1970.

[5] H. Iwaniec and E. Kowalski. Analytic number theory. American Mathematical Society Colloquium Publications. American Mathematical Society, Providence, RI, 2004. xii+615 pp.

[6] J. Kaczorowski and A. Perelli. A new form of the Riemann-van Mangold explicit formula. Boll. Un. Mat. Ital. B (7), 10(1):51-66, 1996.

[7] Jianya Liu and Yangbo Ye. Perron's formula and the prime number theorem for automorphic L-functions. Pure Appl. Math. Q., 3(2, Special Issue: In honor of Leon Simon. Part 1):481-497, 2007.

[8] D. Wolke. On the explicit formula of Riemann-von Mangoldt, II. J. London Math. Soc., 2(28), 1983.

HBNi/Harish-Chandra Research Institute, Jhunsi,, Allahabad -211 019, India., Suri@Hri.res.in

CnRS/Institut de Mathématiques de Marseille, Aix Marseille Université, Centrale Marseille, Site Sud, Campus de Luminy, Case 907, 13288 Marseille Cedex 9, France., OLIVIER.RAMARE@UNIV-AMU.FR 